\title{
Spontaneous recovery of instrumental discriminative responding
}

\author{
ROBERT A. RESCORLA \\ University of Pennsylvania, Philadelphia, Pennsylvania
}

\begin{abstract}
In six experiments, rats received discriminative training in which making a response (R) during a stimulus (S) produced a particular outcome (O). In Experiment 1, that outcome was replaced by a second outcome and responding was tested either immediately or after a delay. More substantial responding was observed with the delayed test. In Experiment 2, a test of transfer to new responses suggested that the growth in performance was not attributable to greater use of particular $\mathrm{S}-\mathrm{O}$ associations. However, in Experiment 3, the growth in responding was found to be specific to particular S-R combinations. Experiment 4 replicated that specificity and demonstrated the importance of using two different outcomes for obtaining the growth in responding with time. Experiments 5 and 6 repeated these observations for the case of extinction, in which $O$ was replaced by nonreinforcement. These results are interpreted as suggesting that an outcome-independent inhibitory S-R process develops, both with extinction and with the use of a second outcome, but dissipates with time.
\end{abstract}

When a stimulus or a response is first followed by a reinforcing outcome and then presented without that outcome, the performance deteriorates. This extinction of responding is a property of virtually all instances of associative learning; consequently, the analysis of its basis has attracted considerable attention. One property of extinction that has seemed especially informative is spontaneous recovery, the partial return of performance with the passage of time after the extinction treatment (e.g., Mackintosh, 1974; Pavlov, 1927). This phenomenon has been routinely interpreted as suggesting that extinction leaves in place at least a portion of the original learning, with the implication that performance decrement involves some process other than removal of that learning.

Recently, Rescorla $(1996,1997)$ has reported a phenomenon like spontaneous recovery with a procedure in which either a stimulus or a response is first followed by one outcome $(\mathrm{Ol})$ and then by another, equivalently valued, outcome $(\mathrm{O} 2)$. One may think of such a shift from $\mathrm{O} 1$ to $\mathrm{O} 2$ as a procedure that provides the conditions for extinction of the associations involving $\mathrm{O} 1$. Yet, because $\mathrm{O} 1$ and $\mathrm{O} 2$ are each capable of serving as a reinforcer, the shift ensures the maintenance of behavior. Under those circumstances, Rescorla observed that performance improved with the passage of time after the use of O2. This was true both in the case of Pavlovian conditioning, when the outcomes were paired with an antecedent stimulus (S), and in the case of instrumental training when they were used to reinforce an instrumen-

This research was supported by National Science Foundation Grant IBN94-04676. Correspondence should be addressed to R. A. Rescorla, Department of Psychology, University of Pennsylvania, 3815 Walnut St., Philadelphia, PA 19104 (e-mail: rescorla@psych.upenn.edu) tal response $(\mathrm{R})$. One interpretation of these findings is that the depressive process involved in extinction also occurs with the shift from one outcome to another but that there is recovery from its effects with the passage of time.

This interpretation is consistent with other observations suggesting that shifting from $\mathrm{O} 1$ to $\mathrm{O} 2$ not only establishes new associations with $\mathrm{O} 2$ but also leaves well preserved the associations with $\mathrm{O} 1$. Experimental techniques that allow one to measure separately the strengths of associations with various outcomes, such as the selective devaluation of an outcome (e.g., Rescorla, 1995) or transfer based on shared outcomes (e.g., Rescorla, 1991), suggest excellent maintenance of associations with both $\mathrm{O} 1$ and $\mathrm{O} 2$. Similar observations have been made about the state of associations with $\mathrm{O} 1$ in the case of extinction, where nonreinforcement is used instead of $\mathrm{O} 2$. The presence of both associations, coupled with the observation that performance is simply maintained at a moderate level when $\mathrm{O} 1$ is replaced by $\mathrm{O} 2$, suggests that some depressive process is undermining performance at the same time as a new association with $\mathrm{O} 2$ is being established. If this process were the same as that responsible for the decrement observed in extinction, then the recovery observed with the passage of time after administration of the second outcome would be understandable.

The goal of the present experiments was twofold. First, the intention was to replicate the recovery observed with time after the shift between outcomes using a different paradigm, instrumental discriminative performance. In previous experiments, this observation has been made for simple nondiscriminative instrumental learning in which a response produces the outcomes and simple Pavlovian conditioning in which a stimulus signals those outcomes. It is therefore natural to ask whether a similar observation can be made for a more complex case in which a stimulus signals when the response will yield the outcomes. 


\begin{tabular}{l|c|c|c|c|c} 
Pretrain & Phase 1 & Phase 20 & Delay & Phase 2b & Test \\
\hline R1-01 & S1: Rc-01 & S1: Re-02 & & & S1: Rc \\
R2-02 & S2: Rc-02 & & & S2: Rc-01 & S2: Rc
\end{tabular}

Figure 1. Experimental design of Experiment 1. Initially, two responses, R1 and R2, were trained using different outcomes, $\mathrm{O} 1$ and $\mathrm{O} 2$. Then a third response, $R_{c}$, was reinforced with those different reinforcers in the presence of two stimuli, $\mathbf{S 1}$ and $\mathbf{S 2}$, respectively. Each stimulus signaled the replacement of one outcome by the other either before (Phase 2a) or after (Phase 2b) a 5-day delay. Then, both stimuli were tested with $\mathbf{R}_{\mathrm{c}}$.

Second, the intention was to explore several alternative mechanisms that might be responsible for the increase in responding with time. One possibility is that there are variations in the associations that are retrieved over the course of such experiments (e.g., Bouton, 1991; Spear, 1978). For instance, replacing $\mathrm{O} 1$ either by $\mathrm{O} 2$ or by nonreinforcement could result in the temporary decrease in the likelihood of retrieving associations involving O1. Such selective retrieval favoring the second event could yield behavior depression (in the case of extinction) or maintained behavior (in the case of O2) while allowing preservation of the (nonretrieved) associations with $\mathrm{Ol}$. With the passage of time, a better balance might be restored in the relative likelihood of retrieving the various associations. Such a mechanism would naturally account for the restoration of behavior with the passage of time after extinction. A similar description can be given when $\mathrm{O} 1$ is replaced by $\mathrm{O} 2$, although some additional assumptions may be needed to account for the improved performance when one, rather than another, of two equivalently valued outcomes is retrieved.

A second possibility is that extinction and the use of $\mathrm{O} 2$ each result in a superimposed inhibitory process that is independent of any particular outcome. For instance, Rescorla (1993), following Colwill (1991), has suggested that extinction might result in the superimposition of an inhibitory stimulus-response ( $\mathrm{S}-\mathrm{R}$ ) association that depresses performance while leaving any outcome-based associations intact. Spontaneous recovery would then result from the partial loss of that inhibition with the passage of time.

In the present experiments, standard discriminative instrumental training procedures with rat subjects were used to explore these possibilities. Experiment 1 provided a demonstration of an increase in the degree to which a discriminative stimulus controls responding with time after reinforcement with a second outcome. Experiment 2 directly assessed the changes that take place in the associations with the $\mathrm{O} 1$ and $\mathrm{O} 2$ with the passage of time, to test whether such changes might have accounted for the change in performance observed in Experiment 1. Experiment 3 examined the degree to which the performance change was specific to particular $\mathrm{S} R$ combinations. Ex- periment 4 examined the importance of $\mathrm{O} 1$ and $\mathrm{O} 2$ being different outcomes. Experiments 5 and 6 explored the same questions for the case of extinction.

\section{EXPERIMENT 1}

In Experiment 1, rats were trained to make an instrumental response (nose poke) in the presence of two different discriminative stimuli for two different outcomes. Then, each stimulus had its original outcome replaced with the alternative outcome. For one stimulus, that replacement was conducted immediately before a final test; for the other stimulus, a delay intervened before the test.

Figure 1 schematizes the experimental design in more detail. During pretraining, all animals were trained to make two responses, R1 and R2, each earning different outcomes, $\mathrm{O} 1$ and $\mathrm{O} 2$. These responses were not used further in the present experiment, but they played a role in the companion Experiment 2. During Phase 1, two stimuli - a light and a noise - counterbalanced as S1 and $\mathrm{S} 2$, signaled that a common nose-poke response $\left(\mathrm{R}_{\mathrm{c}}\right)$ would lead to either a solid pellet or a liquid sucrose, counterbalanced as $\mathrm{O} 1$ and $\mathrm{O} 2$. Then, one of the stimuli, S1, had its outcome switched before a 5-day interval, and the other, S2, had its outcome switched after that interval. Finally, both stimuli were tested with $R_{c}$ nonreinforced in their presence. The result was that $S 1$ was tested after a delay and S2 was tested immediately after reinforcement with the alternative outcomes. Hence, S1 had the opportunity for recovery from any depressive effects of Phase 2, whereas S2 did not.

The particular spontaneous recovery design used here is like that employed in previous experiments from this lab but deviates from the conventional procedure. The usual design would switch reinforcers (or conduct extinction) for both $\mathrm{S} 1$ and S2 immediately after original training but then test one stimulus immediately and one after a delay. Indeed, in many experiments, responding is compared for the same stimulus at the end of its treatment and then some time later. However, those procedures test the stimuli at different times in the life of the animal, thereby confounding any general changes in performance with the recovery process under investigation. 
Moreover, they confound the time since initial training with the time since a switch in the reinforcer. Consequently, we have adopted the present procedure, which ensures that the stimuli to be compared are tested in the same session and at the same distance from original training.

\section{Method}

\section{Subjects and Apparatus}

The subjects were 16 male Sprague-Dawley rats about 90 days old. They were housed in individual cages and maintained on a food-deprivation regime that kept them at $80 \%$ of their ad-lib body we1ght. They had free access to water in the home cage.

The apparatus consisted of eight operant chambers, measuring $22.9 \times 20.3 \times 20.3 \mathrm{~cm}$, identical to those described in previous reports (e.g., Colwill \& Rescorla, 1985). The two end walls of each chamber were aluminum; the side walls and ceiling were clear Plexiglas. Each chamber had a recessed food magazine in the center of one end wall. A small metal cup, measuring $1.25 \mathrm{~cm}$ in diameter and $1.5 \mathrm{~cm}$ deep, was sunk in the floor of each food magazine. To the left of the magazine was a lever and to the right was a chain suspended from a microswitch mounted on the lid of the chamber. Located directly above the food magazine was a $2-\mathrm{cm}$ opening, behind which was an aluminum plate that activated an attached microswitch when displaced by a nose poke. Inserted under the grid floor, just to the right of the magazine aperture, was a flat metal rod, one end of which was bent back to form a handle. An upward pull on this handle operated a microswitch. Access to these manipulanda could be blocked by covering the lever with a metal shield, retracting the chain through a hole in the ceiling, covering the nosepoke opening with a jeweled lens, and removing the handle pull from under the grid floor. The floor of the chamber was composed of $0.48-\mathrm{cm}$ stainless steel rods, spaced $1.9 \mathrm{~cm}$ apart. Each chamber was enclosed in a sound- and light-resistant shell. Mounted on the inside wall of this shell were speakers that permitted the presentation of a white noise and an $1800-\mathrm{Hz}$ tone, each measuring approximately $76 \mathrm{~dB}$ re $20 \mu \mathrm{N} / \mathrm{m}^{2}$ against a background level of $62 \mathrm{~dB}$. Also mounted on that wall was a $6-\mathrm{W}$ bulb that could be illuminated to provide a light stimulus during the otherwise dark session. The outside celling of the shell supported a solenoid-operated gravity feed valve that was connected via plastic tubing to the cup in the food magazine. This system permitted the presentation of $0.3 \mathrm{ml}$ of an $8 \%$ sucrose solution. Also attached to that food magazine was a dispenser containıng 45-mg pellets (P.J. Noyes Co., Formula A).

Experimental events were controlled and recorded automatically by relays and microprocessors located in an adjoining room.

\section{Procedure}

Initial training. On the 1 st day, the animals received two 20-min magazıne traıning sessions, the first containıng 20 pellet deliveries and the second containing 20 sucrose deliveries. Over the next 2 days, all animals were trained to lever press and chain pull. Each training session allowed responding to earn 25 deliveries of the appropriate outcome on a continuous reinforcement schedule. For half of the animals, lever pressing led to pellets in one session, and chain pulling led to sucrose in the other session; for the other half of the animals, the contingencies were reversed. On the next 2 days, all anımals were trained to nose poke. In the first session, they earned 25 pellets; in the second, they earned 25 sucrose deliveries. On the next day, all animals received two 20-min sessions, durnng each of which the nose poking was reinforced on a variable interval (VI) 60 -sec schedule. In one session, the outcome was pellets; in the other, it was sucrose. Throughout this initial trainıng, individual shaping was used if necessary for a particular response-outcome ( $R$ O) combination.

Over the next 5 days, the animals received VI-60 sec training with both the lever press and the chain pull. The $\mathrm{R}-\mathrm{O}$ associations trained were the same as those given during initial training. Each day, the animals received two 20 -min sessions, one with each $\mathrm{R}-\mathrm{O}$ combination. Although these responses were not used further in the present experiment, they were trained in order to match the procedure of Experiment 1 with that of Experiment 2, in which transfer responses would be required.

Phase 1 discrimination training. On each of the next 14 days, the animals received discrimination training sessions with nose poke. Each session contained 16 presentations each of a $30-\mathrm{sec}$ light and a $30-\mathrm{sec}$ noise. During these stimuli, nose poking resulted in reinforcements according to a VI $30-\mathrm{sec}$ schedule. For half of the animals, responding durıng the noise led to pellets and responding during the light led to sucrose; for the other half of the animals, the outcomes were reversed. The stimulus outcome ( $\mathrm{S} O$ ) combinations were arranged to be orthogonal to the $\mathrm{R}-\mathrm{O}$ assignments used for lever and chain. The intertrial intervals (ITIs) were variable around means of 30,60 , and $90 \mathrm{sec}$ for the first 3 days of training, respectively. Thereafter, the mean ITI was $90 \mathrm{sec}$.

Phase 2a discrimination. On the next 2 days, all animals had discrimination training of the same sort except that, for half of the animals, all 32 stimulus presentations were of the noise, and, for the other half of the animals, all 32 were of the light. The reinforcer was the alternative to the one used with that stimulus in Phase 1. All trials were $30 \mathrm{sec}$ long, and the ITI was variable around a mean of $90 \mathrm{sec}$.

Delay. On the next 5 days, no sessions were conducted. The animals remained in their home cages and were maintained on their deprivation schedule.

Phase $2 b$ discrimination. On the next 2 days, all animals received discrimination sessions like those of Phase $2 \mathrm{a}$, except that the other stimulus and the other outcome occurred.

Test. The test consisted of four nonreinforced presentations each of light and noise. These were appended to the end of the second Phase $2 b$ discrimination session without interruption. The differential responding during this test, as a function of the relative recency of treatment of each stimulus, constituted the data of interest.

\section{Results and Discussion}

Initial training of the responses was without incident. At the end of Phase 1 discrimination training, the mean responses per minute were 49.1 and 8.4 during the discriminative stimuli and the $30-\mathrm{sec}$ pretrial period, respectively. There were no reliable differences as a function of stimulus or outcome identity.

During Phase 2, response rates continued at about the same level. For the stimuli trained immediately after Phase 1 , in Phase 2a, the mean responses per minute were 49.3 and 10.6 during the stimulus and prestimulus periods, respectively. The comparable numbers for the stimuli trained after a 5-day delay in Phase 2 b were 43.2 and 11.3 , respectively.

The data of most interest, from the nonreinforced testing of the light and noise at the end of Phase $2 \mathrm{~b}$, are shown in Figure 2. That figure displays the mean response rate during the 30 -sec period prior to any stimulus delivery and during the stimulus that had been given training with the second outcome either before (in Phase 2a) or after (in Phase 2b) the delay. Not surprisingly, both stimuli continued to elevate responding relative to the prestimulus period. However, there was substantially greater elevation for the stimulus that received that training during Phase 2a [Wilcoxon $T(16)=19, p<.01$ ] 


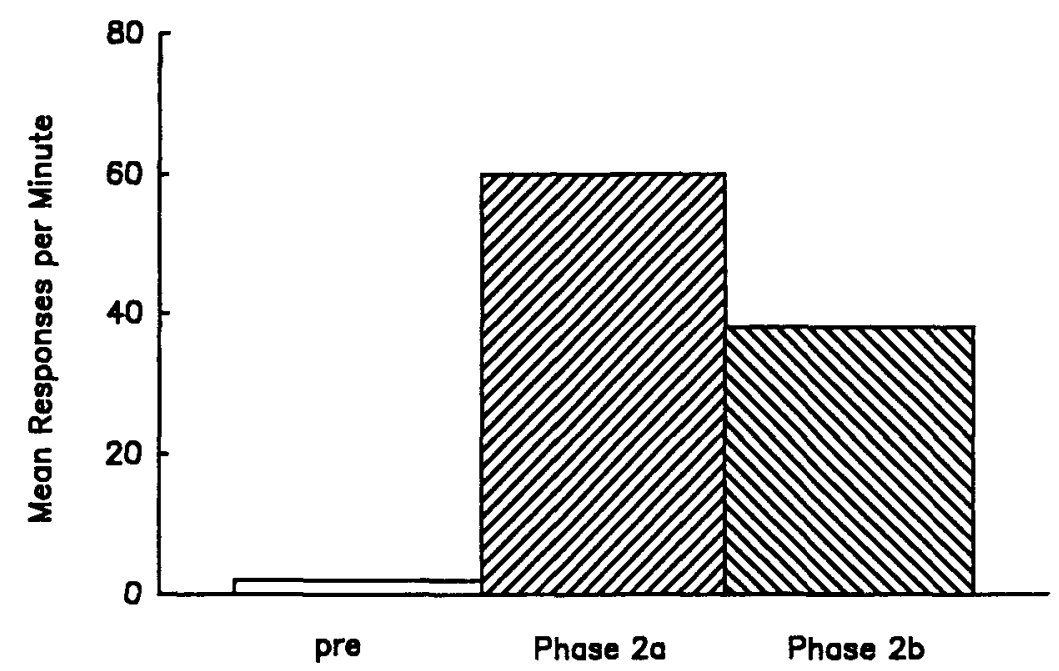

Figure 2. Mean responses per minute in the test of Experiment 1. Responding is shown in the prestimulus period and during a stimulus with which the response had been trained in Phase $2 \mathrm{a}$ or Phase $2 \mathrm{~b}$.

These results replicate, for instrumental discriminative stimuli, the findings previously reported for Pavlovian conditioned stimuli (CSs) and nondiscriminated instrumental responses. Responding was more substantial if a delay intervened after training with a second outcome, relative to responding with the absence of such a delay. The emergence of greater responding only with the passage of time suggests the presence of some depressive process that dissipates. These findings are thus consistent with the view that both the Phase 1 and the Phase 2 associations remain but are undermined by some temporary depressive process.

It is then of interest to explore the nature of that depressive process. Experiment 2 examined the possibility that there is a temporary suppression of retrieval of the association with original $\mathrm{O} 1$ that recovers with time. Experiment 3 examined the alternative that there is a depressive $\mathbf{S}-\mathrm{R}$ process that does not encode the outcomes at all.

\section{EXPERIMENT 2}

The intention of Experiment 2 was to assess the degree to which each stimulus uses its association with a particular outcome at those times when Experiment 1 showed evidence of differential responding. According to a retrieval view, one might expect the association with $\mathrm{O} 2$ to be dominant over that with $\mathrm{O} 1$ immediately after training in Phase 2, with the association involving $\mathrm{O} 1$ reasserting itself over time. That reassertion would then be responsible for the increased overall performance during a stimulus.

The assessment of the individual $\mathrm{O} 1$ and $\mathrm{O} 2$ associations was accomplished by a transfer technique in which the ability of a stimulus to augment a new response is dependent on the strength of their shared associations with a common outcome. This technique has been widely used to measure the strength of associations with outcomes. For instance, Colwill and Rescorla (1988) used initial training procedures quite similar to those employed in Experiment 1 . They trained rats to make two different responses, $\mathrm{R} 1$ and $\mathrm{R} 2$, each earning a different outcome, $\mathrm{O} 1$ and $\mathrm{O} 2$, respectively. Then, they carried out discrimination training in which a light and a noise each signaled that a common third response, $R_{c}$, was reinforced by those same outcomes, but with each stimulus signaling the availability of a particular outcome. When they then presented the stimuli with $\mathrm{R} 1$ and R2 available, they found that each stimulus augmented primarily the transfer response with which it shared an outcome. Thus, the degree of such selective augmentation can be used as a measure of the current strength of the association of a stimulus with each outcome.

Consequently, Experiment 2 was a replication of Experiment 1 , except for the procedure used in the test. The rats were first given training in which lever and chain earned different outcomes, pellet or sucrose. Then, they received discrimination training in which the light and noise signaled the availability of pellet or sucrose for making a nose-poke response in their presence. The outcomes for the nose poke were reversed in Phase 2a for one stimulus and Phase $2 b$ for the other. Immediately after the 2 nd day of Phase $2 b$, the animals were removed from the chamber, and the nose poke was replaced by the R1 and R2, lever and chain. Both stimuli were then presented, with $R 1$ and $R 2$ present. Selective retrieval of the Phase 2 outcome association would anticipate greater evidence of that association for the stimuli tested immediately after training.

\section{Method}

\section{Subjects and Apparatus}

The apparatus was the same as that used in Experiment 1. The subjects were 16 naive male rats of the same stock and maintained in the same manner as in Experiment 1. 


\section{Procedure}

The procedure was identical to that of Experiment 1, with the exception of the test phase. Immediately after the completion of the second session of Phase $2 b$, the nose-poke manipulandum was covered, and the lever and the chain were exposed. The animals were then given four nonrenforced presentations of a $30-\mathrm{sec}$ light and a 30 -sec noise, spaced $30 \mathrm{sec}$ apart.

\section{Results and Discussion}

Initial response and discrimination training proceeded as in Experiment 1. On the final day of the training of the transfer response, the mean rate of responding was 10.5 per minute. On the final day of Phase 1 discrimination training, the mean responses per minute were 35.1 and 6.1 during the stimulus and prestimulus periods, respectively. The comparable numbers were 31.6 and 7.4 on the 2nd day of Phase $2 \mathrm{a}$; they were 33.4 and 5.3 on the 2 nd day of Phase $2 b$.

The data of most interest, from the test, are shown in Figure 3, which displays the mean rate of responding prior to any stimulus presentation and during the stimuli trained with the second outcome in Phase $2 a$ and Phase $2 b$. In each case, responses are separated according to whether they had earned the same outcome as that which the stimulus had signaled in Phase 1 or Phase 2 training. The data suggest that each stimulus augmented responding based on both its Phase 1 outcome and its Phase 2 outcome. Moreover, the degree of augmentation was more substantial when based on the original Phase 1 outcome both for the stimulus trained in Phase $2 a$ and for that trained in Phase $2 b$. This result is not surprising, since each stimulus had received substantially greater training with its Phase 1 outcome. Of most interest, the pattern of eleva- tions was virtually identical for the stimuli receiving their second outcome in Phase $2 a$ and in Phase $2 b$. That is, despite their differential opportunities for recovery since their Phase 2 training, the stimuli were similar in the degree to which they controlled responding based on the Phase 1 and Phase 2 outcomes.

These observations are supported by statistical analysis. There was no reliable difference in responding between the stimuli trained in Phases $2 a$ and $2 b$, either in terms of the individual response rates $[T \mathrm{~s}(15)>46, \mathrm{n} . \mathrm{s}$.] or in terms of the difference between those rates $[T(15)=$ 55 , n.s.]. However, together, the stimuli showed higher rates for the response with which they shared their Phase 1 outcome than that with which they shared their Phase 2 outcome $[T(16)=20, p<.02]$. Responding during the latter stimulus was greater than that during the prestimulus period $[T(16)=27, p<.05]$.

These results show no evidence for differential use of the Phase 1 and Phase 2 outcomes at different points following that training. There is no indication that the Phase 1 outcome was underutilized immediately after Phase 2 training but then showed recovery of use. Since this similarity in use of the different outcomes in a transfer test occurred under conditions identical to those under which overall response rate recovered in Experiment 1, this finding suggests that changes in retrieval of outcome associations might not account for that effect.

It is worth noting that the details of how a retrieval notion might have accounted for the effects of Experiment 1 are, in any case, somewhat unclear. Even if the passage of time resulted in a differential balance in the retrieval of the first- and second-earned outcomes, it does not follow that this should produce augmented be-

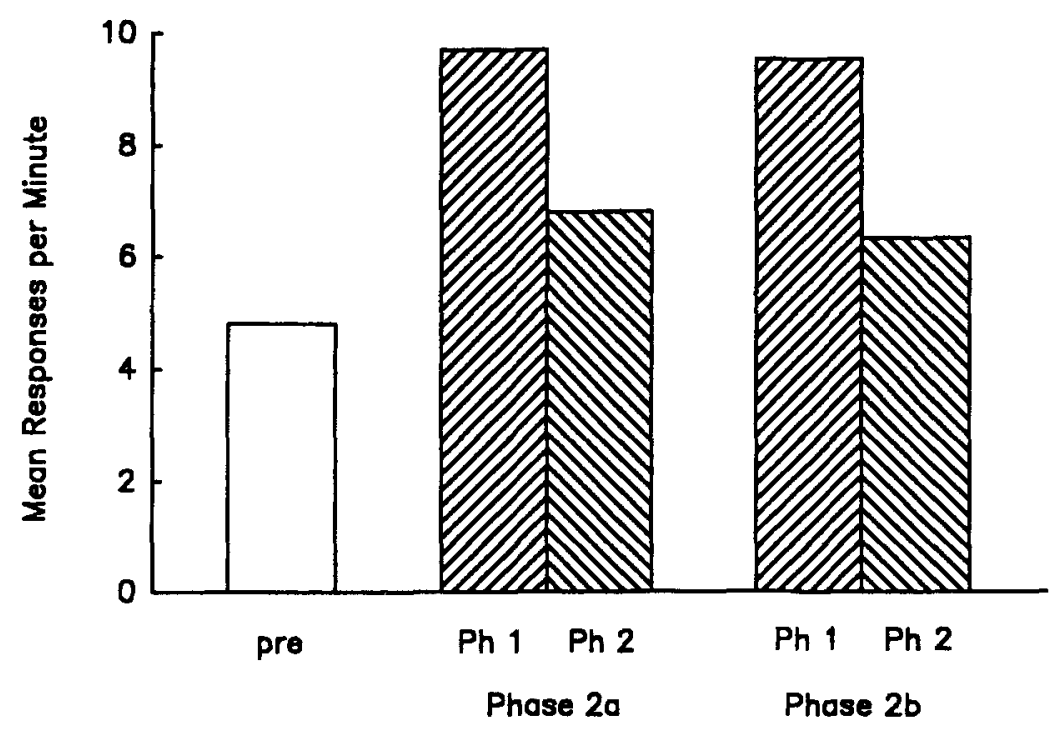

Figure 3. Mean responses per minute in the test of Experiment 2. Responding is shown in a prestimulus period and then during a stimulus treated in Phase $2 \mathrm{a}$ or Phase $2 b$, separated for responses which shared the outcome with that stimulus in its Phase 1 or Phase 2 training. 


\begin{tabular}{r|c|c|c|c} 
Phose 1 & Phase 20 & Delay & Phase 2b & Test \\
\hline S1: $\begin{array}{l}\text { R1-01 } \\
\text { R2-01 }\end{array}$ & $\begin{array}{l}\text { S1: R1-02 } \\
\text { S2: R2-02 }\end{array}$ & & & \\
S2: $\begin{array}{l}\text { R1-01 } \\
\text { R2-01 }\end{array}$ & & & S1: R2-02 & S2: R1, R2 \\
& & & S2: R1-02 &
\end{tabular}

Figure 4. Experimental design of Experiment 3. Each of two responses, $R 1$ and $R 2$, was reinforced by $O 1$ in the presence of each of two stimuli, S1 and S2. Then, two S-R combinations received the alternative outcome, $O 2$, in Phase $2 a$, whereas the other two $S-R$ combinations received that outcome after a delay in Phase $2 b$. All four $S-R$ combinations were tested.

havior. Since the two outcomes are equivalently valued, one might not expect the substitution of retrieval of one for the other to make much difference.

The present findings suggest that there is no growth in the use of the different outcome-based associations with time, at least as measured by a transfer test. Instead, the process that is keeping responding low at the end of Phase 2 but then allows recovery may be outcome-independent. One candidate is the formation of an inhibitory $S-R$ association during Phase 2. Rescorla (1993) has argued that the fact that outcome-based associations remain very effective after extinction suggests that some outcomeindependent depressive process produces the deterioration in performance. It may be that a similar process occurs when there is successive training with two outcomes. Experiment 3 explored that possibility.

\section{EXPERIMENT 3}

The intention of Experiment 3 was to assess whether there is a component to the recovery observed in Experiment 1 that is specific to particular S-R combinations, but independent of associations with the outcomes. For this purpose, a somewhat more complex design, as schematized in Figure 4, was used.

Initially, rats were trained with two different responses, $\mathrm{R} 1$ and $\mathrm{R} 2$, both reinforced by $\mathrm{O} 1$ in the presence of each of two stimuli, S1 and S2. Then in Phase 2a, O2 was substituted as the outcome for two of the $S-R$ combinations, S1-R1 and S2 R2. Then, after a delay of 5 days, O2 was substituted for $\mathrm{O} 1$ as the outcome for the other two $S-R$ pairs, S1-R2 and S2-R1. Immediately thereafter, all four $\mathrm{S}-\mathrm{R}$ combinations were tested. In this design, both stimuli and both responses have been treated with $\mathrm{O} 2$ in both Phase 2a and Phase 2b. Moreover, all of the S-R combinations have undergone training with $\mathrm{O} 2$ at some point. However, if that training results not only in learning about $\mathrm{O} 2$ but also in a depressive process that is specific to combinations of stimuli and responses, then $\mathrm{S} 1-\mathrm{R} 1$ and $\mathrm{S} 2-\mathrm{R} 2$ received that depression in Phase $2 a$, whereas $S 1-R 2$ and S2-R1 had it in Phase 2b. Therefore, the S1-R1 and S2-R2 combinations have had the opportunity to recover prior to the test, whereas the S1-R2 and S2-R1 combinations have not. Consequently, in the final test, responding should be greater to the former combinations. Since the same individual stimuli and responses, as well as the same $\mathrm{O} 2$, were involved in both Phase $2 a$ and Phase $2 b$, any differential recovery could not be attributable to changes in the status of any of those individual components.

\section{Method}

\section{Subjects and Apparatus}

The apparatus was the same as that used in Experiment 1. The subjects were 16 naive male rats of the same stock and maintanned in the same manner as in Experıment 1.

\section{Procedure}

Initial training. The anımals received inıtıal magazıne trainıng with pellets and sucrose in the manner of Experiment 1. They were then trained to both lever press and chain pull using the procedure of Experiment 1. For half of the animals, both responses earned pellets; for the other half of the animals, the responses earned sucrose.

Phase 1 discrimination training. On each of the next 14 days, the animals received two discrimination training sessions, one with lever and one with chain. The sessions were spaced about $1 \mathrm{~h}$ apart. Each session contained 16 presentations each of a 30 -sec light and a 30-sec noise. During these stimul, responding resulted in reinforcements according to a VI $30-\mathrm{sec}$ schedule. The outcome earned was the same as that used in initial training. The ITIs were variable around means of 30,60 , and $90 \mathrm{sec}$ for the first 3 days of training, respectively. Thereafter, the mean ITI was $90 \mathrm{sec}$.

Phase 2 a discrimination. On the next 2 days, all animals continued to receive two discrimination sessions, one with each response. However, one stimulus was presented on all 32 trials of a session, and the outcome used was the alternative to that used in Phase 1. During one session, the stimulus was a light; during the other, it was a noise. Half of the anımals had lever trained during light and the chain trained during noise, whereas the other half had the reverse combinations. These treatments were arranged to be orthogonal to those of the previous stage. All trials were $30 \mathrm{sec}$ long, and the ITI was variable around a mean of $90 \mathrm{sec}$.

Delay. On the next 5 days, no sessions were conducted. The animals remained in their home cages and were maintained on their deprivation schedule.

Phase 2b discrimination. On each of the next 2 days, all an1mals received two discrimination sessions, one with lever and one with chain. Each response was reinforced with the same outcome as that used in Phase 2a. However, that reinforcement took place in the presence of the alternatıve stımulus.

Test. Immediately after the completion of the second session of Phase $2 b$, all animals received a test session, with both lever and chain present. In that session, they received four nonreinforced 30 sec presentations of each stimulus. The question of interest is the 


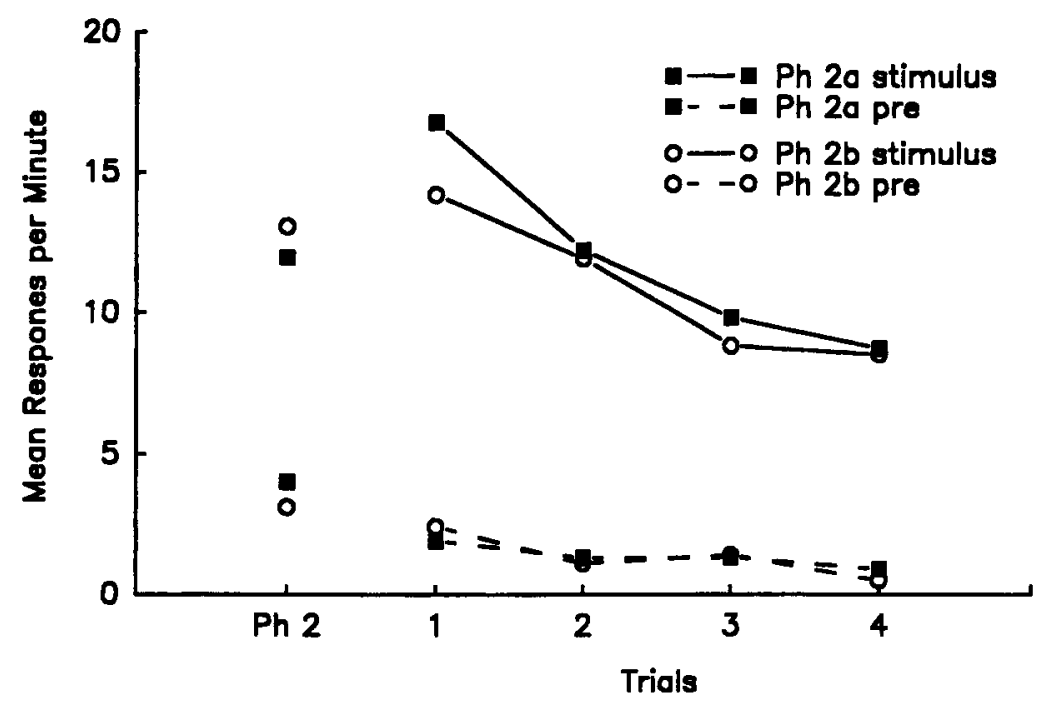

Figure 5. Results of Experiment 3. Responding is shown at the end of Phase 2 and over the course of an extinction test during the prestimulus and stimulus periods for the $\mathbf{S}-\mathbf{R}$ combination that had been treated in Phase $2 a$ or Phase $\mathbf{2 b}$.

magnitude of responding for each $\mathrm{S}-\mathrm{R}$ combination as a function of whether that combination had been trained in Phase $2 \mathrm{a}$ or Phase $2 \mathrm{~b}$.

\section{Results and Discussion}

Initial response training and discrimination learning proceeded smoothly. On the final day of Phase 1 training, the mean responses per minute were 12.4 and 1.8 during the stimulus and prestimulus periods, respectively. The lower response rates observed with lever and chain, compared with those with nose poke in Experiments 1 and 2, are typical for this laboratory. The response rates remained at about the same level during Phase 2. The mean responses per minute during Phase $2 \mathrm{a}$ were 12.0 and 4.0 during the stimulus and prestimulus periods; the comparable figures for Phase $2 b$ were 13.1 and 3.1 , respectively.

The data of most interest, from the test of both stimuli with both responses, are shown in Figure 5, which displays the mean responses per minute during the stimulus and prestimulus periods for the S-R combinations that had been treated in Phase $2 \mathrm{a}$ and Phase $2 \mathrm{~b}$. Responding was, not surprisingly, greater during all of the stimulus periods. However, that superiority was greater for the combinations treated in Phase 2a than for those treated in Phase $2 b$. Responding declined over the course of the extinction test, and the difference was greatest in the first test period. During that period, the superiority of the Phase 2 a combinations was reliable $[T(16)=24, p<.02]$.

These results support the proposition that at least a portion of the change in responding over time is specific to particular combinations of stimuli and responses, but not to either of them individually or to the outcomes. One natural interpretation is that Phase 2 involved a depressive $\mathrm{S}-\mathrm{R}$ process that recovers with time.

\section{EXPERIMENT 4}

Experiment 4 had two intentions. The first was to assess the importance of using a new outcome, O2, during Phase 2. Our previous reports of increases in behavior with time when a Pavlovian stimulus or an instrumental response is first followed by $\mathrm{O} 1$ and then by $\mathrm{O} 2$ have found this effect only when $\mathrm{O} 2$ is different from $\mathrm{O} 1$. This finding is relevant to an account in terms of the development of a temporary decremental process. According to such an account, as the association with $\mathrm{O} 2$ begins to develop, the combination of the associations with $\mathrm{O} 2$ and $\mathrm{O} 1$ leads to an inappropriately large total associative strength. That, in turn, forces the development of an inhibitory process. However, if $\mathrm{O} 2$ were identical to $\mathrm{O} 1$, no new association would develop, and no decremental process would result. It is therefore of interest to demonstrate that the growth in discriminative instrumental behavior with time depends on the use of a different $\mathrm{O} 2$, in the same manner as did the comparable growth after simple instrumental training and Pavlovian conditioning.

Second, the magnitude of the effect observed in Experiment 3 was relatively small. It seems likely that this is attributable in part to the complexity of the experimental design, with the opportunities it provides for generalization across $S-R$ pairs. Nevertheless, it would be valuable to replicate the specificity of recovery for particular S-R pairs found in Experiment 3.

Therefore, Experiment 4 was designed to build on the S-R specificity found in Experiment 3 to provide a demonstration of the importance of changing from $\mathrm{Ol}$ to $\mathrm{O} 2$ in observing the growth in responding with time. The experimental design is illustrated in Figure 6. Initially, the animals were trained to make two different in- 


\begin{tabular}{|c|c|c|c|c|}
\hline Phase 1 & Phase 20 & Delay & Phase 2b & Test \\
\hline S1: $\begin{array}{l}R 1-01 \\
R 2-01\end{array}$ & S1: $\begin{array}{l}R 1-02 \\
R 2-01\end{array}$ & & & S1: R1, R2 \\
\hline S2: $\begin{array}{l}\mathrm{R} 1-02 \\
\mathrm{R} 2-02\end{array}$ & & & S2: $\begin{array}{l}R 1-01 \\
R 2-02\end{array}$ & S2: R1, R2 \\
\hline
\end{tabular}

Figure 6. Design of Experiment 4. Each of two responses, R1 and R2, was reinforced in the presence of each of two stimuli, S1 and S2. Then one stimulus, S1, received training with both responses in Phase 2a, and the other stimulus, S2, received training in Phase $2 b$. For one response, R1, the outcomes were changes from Phase 1, whereas for the other response, $R 2$, they were left unchanged. Both responses were then tested during both stimuli.

strumental responses, $\mathrm{R} 1$ and $\mathrm{R} 2$, with both rewarded in the presence of two different stimuli, S1 and S2. Each stimulus signaled the availability of a particular outcome, but, across stimuli, both responses earned both outcomes. Then, in Phase 2a, one of the stimuli, S1, received additional training with both of its responses. For one of the responses, the outcome remained the same as in Phase 1; for the other response, the alternative outcome was used. After a delay, a similar procedure was used for S2, with the outcome again altered for one response but not the other. Finally, both responses were tested in the presence of both stimuli.

The consequence of these treatments is that Sl received its Phase 2 training early, at a distance from the eventual test, whereas S2 had its Phase 2 training later, near the test. However, for R1, the training experiences involved a change of outcome, whereas, for R2, the same outcome was used in Phase 2 as had been employed in Phase 1 . The treatment of $R 1$ constitutes a replication of the procedures of Experiment 1. The treatment of $R 2$ provides a within-subject comparison in which the same outcome is used for Phase 2 and Phase 1 . An observation of a greater ability of S1, compared with $\mathrm{S} 2$, to augment $R 1$, coupled with an absence of such a difference for $R 2$, would both demonstrate the importance of changing the outcome and replicate the kind of S-R interaction observed in Experiment 3.

\section{Method \\ Subjects and Apparatus \\ The apparatus was the same as that used in Experiment 1. The subjects were 16 naive male rats of the same stock and mantained in the same manner as in Experiment 1.}

\section{Procedure}

Initial training. The animals received initial magazine training with pellets and sucrose in the manner of Experiment 1 . They were then trained to both lever press and chain pull using the procedure of Experiment 1. Each response was trained twice, once with sucrose and once with pellets.

Phase 1 discrimination training. On each of the next 12 days, the animals recelved two discrimination traning sessions, one with lever and one with chain. The sessions were spaced about $1 \mathrm{~h}$ apart. Each session contained 16 presentations each of a $30-\mathrm{sec}$ light and a $30-\mathrm{sec}$ noise. During these stımul, responding resulted in rein- forcements according to a VI 30-sec schedule. For half of the animals, responding during the light earned pellets and responding during the noise earned sucrose; for the other half of the anımals, the outcomes were reversed. The ITIs were variable around means of 30,60 , and $90 \mathrm{sec}$ for the first 3 days of training, respectively. Thereafter, the mean ITI was $90 \mathrm{sec}$.

Phase 2a discrimination. On the next 2 days, all animals continued to receive two discrimınation sessions, one with each response. However, one stimulus was presented on all 32 trials of each session. For one response, the outcome used was the same as that in Phase 1; for the other response, the alternative outcome was used. The identity of the stimulus used and the treatment of responses were balanced with regard to prior treatments. All trials were $30 \mathrm{sec}$ long, and the ITI was varrable around a mean of $90 \mathrm{sec}$.

Delay. On the next 5 days, no sessions were conducted. The animals remained in their home cages and were maintained on their deprivation schedule.

Phase 2b discrimination. On each of the next 2 days, all animals received two discrimination sessions, one with lever and one with chain. Each response was reinforced with the alternative outcome from that used in Phase 2a. That reinforcement took place in the presence of the alternative stimulus. As a result of these treatments, each response was trained in both Phase $2 a$ and Phase $2 b$; however, for R1, the outcome was shifted in both phases, whereas, for R2, it was left as in Phase 1.

Test. Immediately after the completion of the second session of Phase $2 b$, all anımals received two test sessions, one each with lever and chain present. In those session, they received four nonreinforced $30-\mathrm{sec}$ presentations of each stimulus.

\section{Results and Discussion}

Initial response training and discrimination learning proceeded smoothly. On the final day of Phase 1 training, the mean responses per minute were 13.8 and 3.3 during the stimuli and prestimulus periods, respectively. The response rates remained at about the same level during Phase 2. The mean responses per minute during Phase $2 \mathrm{a}$ were 13.2 and 3.2 during the stimulus and prestimulus periods; the comparable figures for Phase $2 \mathrm{~b}$ were 13.3 and 4.1 , respectively. There were no reliable differences as a function of treatments.

The data of most interest, from the test of both stimuli with both responses, are shown in Figure 7, which displays the mean responses per minute for the responses that had one outcome in both Phase 2 and Phase 1 or that had two different outcomes in those phases. In each case, responding is shown during the prestimulus period and 


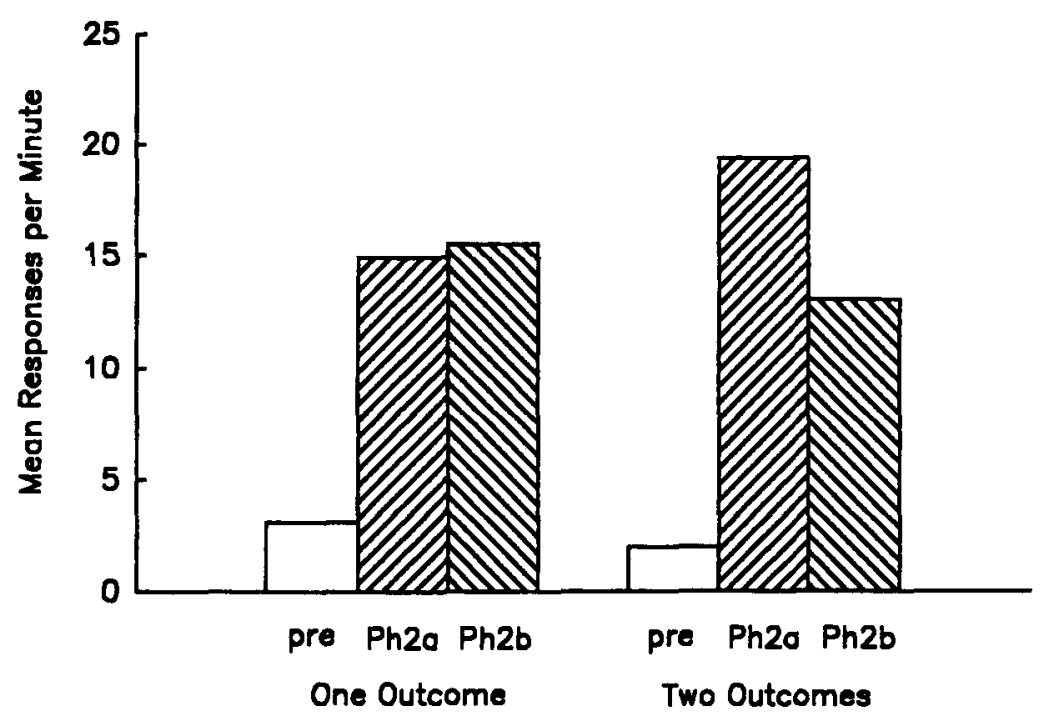

Figure 7. Results of Experiment 4. Responding is shown for two responses, one trained with only one outcome and one with two outcomes. Prior to this test, each response had received training with one stimulus in Phase $2 a$ and one in Phase $2 b$.

during the stimulus used in Phase $2 a$ and Phase $2 b$. Responding was elevated by both the Phase $2 a$ and the Phase $2 b$ stimulus, for both responses. However, for the two-outcome case, the degree of elevation was greater for the Phase 2a stimulus; for the one-outcome case, the elevation was similar for both stimuli. The difference between Phase $2 \mathrm{a}$ and Phase $2 \mathrm{~b}$ stimuli for the two-outcome response proved reliable $[T(16)=27.5, p<.05]$, whereas the difference for the one-outcome response was not $[T(16)=58.5]$. Moreover, the difference in responding to the two stimuli was reliably greater for the two-outcome response, compared with the one-outcome response $[T(15)=23, p<.05]$.

These results suggest that the increased responding with time after the use of a second outcome depends importantly on the identity of that second outcome being different from that used in the prior phase. This result is consistent with the present interpretation in terms of a temporary outcome-independent depressive process developing when the total associative strength is too large. Moreover, because these results were observed in a within-subject design in which the stimuli showed the augmented responding with one response but not another, they repeat the observation of Experiment 3 that the change in responding with time is specific to particular S-R combinations.

\section{EXPERIMENT 5}

A major reason for investigating the fate of $\mathrm{S}-\mathrm{O} 1$ associations when $\mathrm{O} 1$ is replaced by $\mathrm{O} 2$ is that it may shed some light on the case of extinction, where nonreinforcement is used instead of O2. Consequently, it is of interest to explore the same questions examined in Experiments 2 and 3 for the case of extinction. Experi- ment 5 examined the potential differential retrieval of $\mathrm{S}-\mathrm{O}$ associations after the passage of time following extinction. Experiment 6 focused on potential changes in particular S-R combinations with time after extinction.

The procedure of Experiment 5 was parallel to that of Experiment 2. The rats were first given reinforcement of two target transfer responses, lever and chain, with different outcomes. Then, they received discrimination training in which a light and a noise signaled the availability of pellets or sucrose for making other responses, nose poke and handle pull, with each response associated with one stimulus. One of those stimuli then received extinction with its response in Phase $2 \mathrm{a}$, whereas the other received extinction in Phase $2 b$. Two different responses were used with light and noise in order to reduce the likelihood of generalization of extinction between the stimuli. Immediately after the 2 nd day of Phase $2 b$, the animals were given a transfer test in which light and noise were superimposed on lever and chain. It was expected that each stimulus would selectively augment performance of the transfer response with which it shared an outcome. The question of interest was whether that selective augmentation would be stronger for the stimulus extinguished in Phase 2 a than for that extinguished in Phase $2 \mathrm{~b}$. If extinction results in the reduced likelihood of retrieval of the originally trained S-O associations, then any recovery of that retrieval with the passage of time should be evident in the greater transfer for the stimulus extinguished in Phase $2 a$.

\section{Method}

\section{Subjects and Apparatus}

The apparatus was the same as that used in Experiment 1 . The subjects were 16 naive male rats of the same stock and maintained in the same manner as in Experiment 1. 


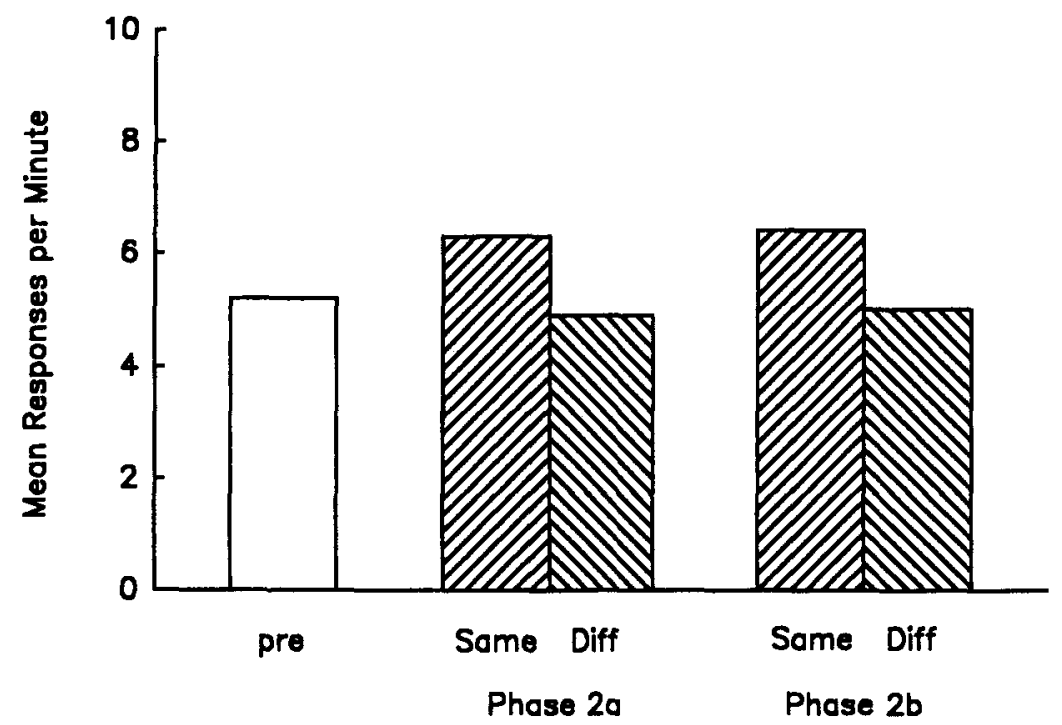

Figure 8. Mean responses per minute during the test of Experiment 5. Responding is shown during the prestimulus and during a stimulus signaling the same or a different outcome for the stimulus extinguished in Phase $2 \mathrm{a}$ and $P$ hase $2 \mathrm{~b}$.

\section{Procedure}

The procedure was identical to that of Experiment 1, with two exceptions. First, extinction replaced training with the alternative outcome in Phase 2. Second, both a nose poke and a handle pull were used as responses during the initial discrimination training. In a counterbalanced fashion, each response was trained with either light or noise. This was accomplished by running two 32 -trial sessions per day, one with each stimulus signaling a particular outcome for making a particular response. Then, one stimulus received 2 days of extinction with its response in Phase 2a, and, after a 5-day interval, the other stimulus received 2 days of extinction with its response in Phase $2 \mathrm{~b}$. Immediately after the completion of the second session of Phase $2 b$, the lever and chain were inserted into the chambers, and the animals were then given four nonreinforced presentations of a 30-sec light and a 30-sec noise, spaced $30 \mathrm{sec}$ apart.

\section{Results and Discussion}

Initial response and discrimination training proceeded smoothly. On the final day of the training of the transfer responses, the mean rate of responding was 9.1 per minute. On the final day of Phase 1 discrimination training, the mean responses per minute were 26.5 and 3.5 during the stimulus and prestimulus periods, respectively. With the institution of extinction, responding rapidly deteriorated. The mean response rates were 0.6 and 0.2 during the stimulus and prestimulus periods in the second half of the 2 nd day of Phase $2 a$. The comparable numbers were 0.5 and 0 during Phase $2 b$.

The data of most interest, from the transfer test, are shown in Figure 8, which displays the mean rate of responding prior to any stimulus presentation and during the stimuli extinguished in Phase $2 \mathrm{a}$ and Phase $2 \mathrm{~b}$. The results are separated according to whether the stimulus signaled the same or a different outcome, compared with that earned by the transfer response. As in previous reports (e.g., Colwill \& Rescorla, 1988), the presentation of a stimulus augmented that response with which it shared an outcome but not that had earned a different outcome. More importantly, that pattern of differential transfer was similar for the stimuli extinguished in Phase $2 \mathrm{a}$ and Phase $2 \mathrm{~b}$. Although there were no reliable differences between those stimuli, each showed a selective augmentation of responding $[T \mathrm{~s}(16)<28, p \mathrm{~s}<.05]$.

The observation of selective augmentation confirms earlier findings that extinction fails to eliminate previously learned $\mathrm{S}-\mathrm{O}$ associations. Despite extinction, each stimulus continued to show evidence of enhancing only those responses with which it shared an outcome. The observation that the magnitude of that augmentation was similar whether the stimulus had been extinguished either a few minutes previously (in Phase $2 b$ ) or 7 days earlier (in Phase 2a) suggests little change in the use of those $\mathrm{S}-\mathrm{O}$ associations with the passage of time since extinction. This seems inconsistent with the view that extinction produced a temporary reduction in the use of an S-O association, from which there was recovery with the passage of time. Instead, in the case of extinction, as in the case of training with a second outcome, the original $\mathrm{S}-\mathrm{O}$ association seems to exhibit little change with the passage of time after the potentially depressive treatment.

It is, of course, possible that the transfer procedure used here is sufficiently sensitive that it can detect the presence of $\mathrm{S}-\mathrm{O}$ associations even under circumstances in which they are reduced in effectiveness in controlling their original response. It might be, for instance, that the transfer test constitutes a change in context, which allows the $\mathrm{S}-\mathrm{O}$ associations to reveal themselves after extinction (cf. Bouton, 1991). However, the results of Experiment 5 are consistent with the view that extinction leaves the $\mathrm{S}-\mathrm{O}$ associations sufficiently intact such that neither changes in their strength nor use over time is responsible for spontaneous recovery. 


\section{EXPERIMENT 6}

Experiment 6 was intended to provide evidence parallel to that of Experiment 3, but for the case of extinction. The design was identical to that of Experiment 3, except that extinction replaced the training with a second outcome in Phase 2. Initially, the animals were trained to lever press and chain pull during light and noise, with a pellet as the reinforcer. Then, two of the four S-R combinations were extinguished in Phase $2 \mathrm{a}$; after an interval of 5 days, the other two $S-R$ combinations were extinguished, and then all four combinations were tested. The issue is the degree to which spontaneous recovery with time is specific to particular $\mathrm{S}-\mathrm{R}$ combinations in the manner expected if extinction involves the development of inhibitory S-R associations.

\section{Method}

\section{Subjects and Apparatus}

The apparatus was the same as that used in Experiment 1. The subjects were 16 naive male rats of the same stock and maintained in the same manner as in Experiment 1.

\section{Procedure}

Initial training. The procedure was modeled closely on that of Experiment 3. The animals recelved initial magazine training with pellets and were then trained to both lever press and chain pull using the procedure of Experiment 1.

Phase 1 discrimination training. On each of the next 14 days, the animals received two discrimination training sessions, one with lever and one with chain. The sessions were spaced about $1 \mathrm{~h}$ apart. Each session contained 16 presentations each of a $30-\mathrm{sec}$ light and a 30-sec noise. During these stimuli, responding resulted in pellets according to a VI $30-\mathrm{sec}$ schedule. The ITIs were variable around means of 30,60 , and $90 \mathrm{sec}$ for the first 3 days of training, respectively. Thereafter, the mean ITI was 90 sec.

Phase 2a extinction. On the next day, all animals continued to receive two sessions, one with each response. However, one stimulus was presented on all 32 trials of a session and was nonrein- forced. During one session, the stimulus was a light; during the other, it was a noise. Half of the animals had lever pressing extinguished during light and the chain pulling extinguished during noise, whereas half had the reverse combinations. All trials were $30 \mathrm{sec}$ long, and the ITI was variable around a mean of $90 \mathrm{sec}$.

Delay. On the next 5 days, no sessions were conducted. The animals remained in their home cages and were maintained on their deprivation schedule.

Phase $2 b$ extinction. On the next day, all anımals received two extinction sessions, one with lever and one with chain. Each response was extinguished as in Phase $2 \mathrm{a}$; however, that extınction took place in the presence of the alternative stimulus.

Test. Immediately after the completion of Phase $2 \mathrm{~b}$, all animals received a test session, with both lever and chain present. In that session, they received four nonreinforced $30-\mathrm{sec}$ presentations of each stimulus. The question of interest is the magnitude of responding for each $\mathrm{S}-\mathrm{R}$ combination as a function of whether that combination had been extinguished in Phase $2 a$ or Phase $2 b$.

\section{Results and Discussion}

Initial response training and discrimination learning proceeded smoothly. On the final day of Phase 1 training, the mean responses per minute were 18.0 and 3.1 during the stimulus and prestimulus periods, respectively. Over the course of Phase 2 , responding deteriorated. On the last block of 8 trails in Phase 2a, the mean response rates were 2.1 and 0.3 during the stimulus and prestimulus periods, respectively. The comparable numbers for Phase $2 \mathrm{~b}$ were 1.6 and 0.2 .

The data of most interest, from the test of both stimuli with both responses, are shown in Figure 9. That figure displays the mean responses per minute during the stimulus and prestimulus periods for the $S-R$ combinations that had been treated in Phase $2 a$ and Phase $2 b$ for the last block of extinction and then over the course of the test. Responding remained low for those $\mathrm{S}-\mathrm{R}$ combinations that underwent extinction in Phase 2b. However, there was substantial recovery for those combinations extinguished

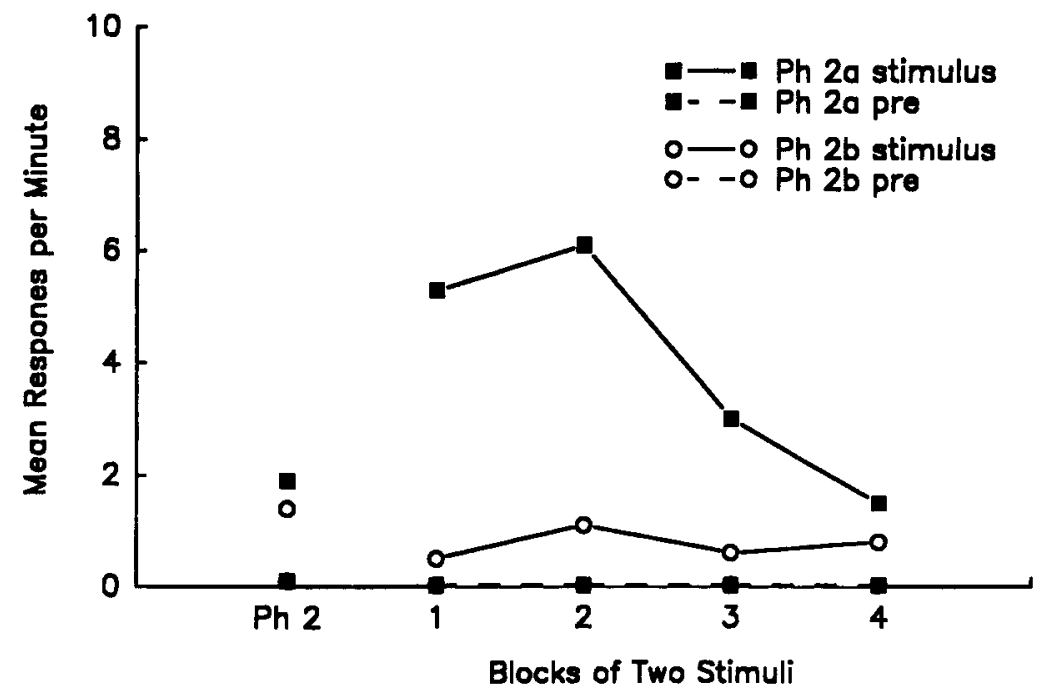

Figure 9. Results of Experiment 6. Responding is shown at the end of Phase 2 and over the course of an extinction test during the prestimulus and stimulus periods for the $\mathbf{S}-\mathbf{R}$ combination that had been treated in Phase 2 a or Phase $\mathbf{2 b}$. 
in Phase 2a. The superiority of responding to the latter combinations was highly reliable $[T(16)=1, p<.01]$.

These results support the proposition that at least a portion of the change in responding over time following extinction is specific to particular combinations of stimuli and response, but not to either of them individually or to the outcomes. One natural interpretation is that Phase 2 involved a depressive $S \mathrm{R}$ process that recovers with time.

\section{GENERAL DISCUSSION}

These experiments show evidence for an increase in responding over time in an instrumental discrimination procedure after successive reinforcement with two outcomes. This confirms for discriminated instrumental learning an observation that has previously been made in simple Pavlovian conditioning and nondiscriminated instrumental learning. These results also suggest that the basis of this increase is not a change in the relative retrieval of the associations involving the first and second outcome but rather some process that is specific to particular S-R combinations. In a similar way, these experiments indicate that the spontaneous recovery that occurs following extinction is specific to particular S-R combinations and does not depend on changes in the retrieval of the originally learned associations.

As with previous preparations, the superior responding after a delay cannot be attributed to a variety of changes in the component stimuli, responses, and outcomes themselves. The observation in Experiments 1, 3, and 4 that the same response occurs with different frequencies during stimuli that differ in the time of delay since their last treatment implies that changes in the response per se are not critical. For instance, one cannot attribute the results to differential response fatigue and recovery from that fatigue. The parallel observation in Experiment 6 suggests the same conclusion for the case of extinction. Similarly, the observation in those experiments of differential performance after different delays for stimuli and responses associated with the same outcomes suggests that changes in the outcome per se are not critical. That is, changes in the value of the outcome, such as local satiation effects, would not produce these results. Apparently, any changes in the status of the outcome representation per se (e.g., Rescorla \& Cunningham, 1978) cannot fully account for the present changes. Finally, the finding in Experiments 3 and 6 that the same stimulus controlled different levels of performance for responses with which its treatment had occurred at different times in the past suggests that changes in stimulus salience (e.g., Robbins, 1990) are not essential.

These results are consistent with the view that the present extinction and outcome-shifting procedures result in the development of some outcome-independent decremental process. Apparently, that process acts to interfere with performance during Phase 2 but produces less interference with the passage of time. The specificity to particular $\mathrm{S}-\mathrm{R}$ combinations encourages an interpreta- tion in terms of the development of inhibitory S-R associations. But, the basis for the change with time remains unclear. On the one hand, such associations might lose strength with time in the manner frequently envisioned for inhibitory processes since Pavlov's (1927) original suggestion. On the other hand, it is possible that what changes with time is the likelihood of retrieval of the inhibitory mechanism. The present results provide no basis for choosing between those alternatives.

As noted earlier, the procedure used here differs from the typically spontaneous recovery design not only in replacing extinction with a second outcome but also in the way that delay is interposed in the experiment. Instead of training with the second outcome immediately after training with the first and then varying the delay until a test, the present experiments held constant the interval between initial training and test but varied the placement of the training with the second outcome in that interval. This has the advantage of allowing testing at a common time of stimuli and response previously treated differently. This is important if one anticipates that there might be changes over time that are unrelated to the treatments. Moreover, it helps to emphasize that the important comparison is between responding at the time of the test for stimuli and responses that have been treated differently at varying times in the past. The more typical comparison between responding at the end of Phase 2 treatment and test clearly confounds recovery with general changes in the animal.

However, the present procedure has its own disadvantages. For instance, the stimulus and response combination that has the shorter delay to test is also the one that was treated farther from initial training and, with the present within-subject designs, trained second. The similarity of responding during Phases $2 \mathrm{a}$ and $2 \mathrm{~b}$ suggests that these are not important determining variables. Although direct comparisons between alternative designs were not conducted for the present case of discriminative instrumental training, they are available for the case of Pavlovian conditioning (Rescorla, 1997). The similarity of the results with different procedures is encouraging, not only for the interpretation of the present data but also for interpretation of the many spontaneous recovery experiments that have preceded it.

In any case, the present data provide evidence for a change like spontaneous recovery after one outcome is replaced by another in instrumental discrimination training. Moreover, they suggest that the recovery lies neither in the individual $\mathrm{S}, \mathrm{R}$, or $\mathrm{O}$ events nor in the $\mathrm{S}-\mathrm{O}$ or $\mathrm{R} \mathrm{O}$ associations. Rather recovery showed a specificity to particular $S-R$ pairs in the manner to be expected if inhibitory S-R associations play an important role.

\section{REFERENCES}

Bouton, M. E. (1991). Context and retrieval in extinction and in other examples of interference in simple associative learning. In $\mathrm{L}$. W. Dachowsk1 \& C. F Flaherty (Eds.), Current topics in animal learning

Brain, emotion, and cognition (pp. 25-53). Hillsdale, NJ Erlbaum. Colwill, R. M. (1991). Negatıve discrimınative stımul provide infor- 
mation about the identity of omitted response-contingent outcomes Animal Learning \& Behavior, 19, 326-336.

Colwill, R. M., \& ResCorla, R. A. (1985). Post-conditioning devaluation of a reinforcer affects instrumental responding. Journal of Experimental Psychology: Animal Behavior Processes, 11, 120-132.

COLWILL, R. M., \& REsCorla, R. A. (1988). Associations between the discriminative stimulus and the reinforcer in instrumental learning. Journal of Experimental Psychology: Animal Behavior Processes, 14, 155-164.

Mackintosh, N. J. (1974). The psychology of animal learning. New York: Academic Press.

Pavlov, I. P. (1927). Conditioned reflexes (G. V. Anrep, Trans.). London: Oxford University Press.

RESCORLA, R. A. (1991). Associations of multiple outcomes with an instrumental response. Journal of Experimental Psychology: Animal Behavior Processes, 17, 465-474.

Rescorla, R. A. (1993). Inhibitory associations between $S$ and $R$ in extinction. Animal Learning \& Behavior, 21, 327-336.

RESCORLA, R. A. (1995). Full preservation of a response-outcome as- sociation through training with a second outcome. Quarterly Journal of Experimental Psychology, 48B, 252-261.

RESCORLA, R. A. (1996). Spontaneous recovery after training with multiple outcomes. Animal Learning \& Behavior, 24, 11-18.

RESCORLA, R. A. (1997). Spontaneous recovery after Pavlovian conditioning with multiple outcomes. Animal Learning \& Behavior, 25, 99-107.

Rescorla, R. A., \& Cunningham, C. L. (1978). Recovery of the US representation over time during extinction. Learning \& Motivation, 9, 373-391.

RoBbiNs, S. J. (1990). Mechanisms underlying spontaneous recovery in autoshaping. Journal of Experimental Psychology: Animal Behavior Processes, 16, 235-249.

SPEAR, N. E. (1978). Processing of memories: Forgetting and retention. Hillsdale, NJ: Erlbaum.

(Manuscript received November 11, 1996; revision accepted for publication March 7, 1997.) 